\title{
Nijboer-Zernike phase retrieval for high contrast imaging
}

\section{Principle, on-sky demonstration with NACO, and perspectives in vector vortex coronagraphy}

\author{
P. Riaud ${ }^{1,2}$, D. Mawet ${ }^{3,4}$, and A. Magette ${ }^{5,2}$ \\ 160 rue des bergers, 75015 Paris, France \\ e-mail: riaud.pierre@gmail.com \\ 2 Université de Liège, 17 Allée du 6 Août, 4000 Sart Tilman, Belgium \\ 3 European Southern Observatory, Alonso de Cordóva 3107, Vitacura, Santiago, Chile \\ e-mail: dmawet@eso.org \\ 4 NASA-Jet Propulsion Laboratory, California Institute of Technology, 4800 Oak Grove Drive, Pasadena, CA 91109, USA \\ 5 Techspace-Aero, route de Liers 121, 4041 Milmort, Belgium \\ e-mail: arnaud.magette@techspace-aero.be
}

Received 16 May 2012 / Accepted 19 July 2012

\begin{abstract}
We introduce a novel phase retrieval method for astronomical applications based on the Nijboer-Zernike (NZ) theory of diffraction. We present a generalized NZ phase retrieval process that is not limited to small and symmetric aberrations and can therefore be directly applied to astronomical imaging instruments. We describe a practical demonstration of this novel method that was recently performed using data taken on-sky with NAOS-CONICA, the adaptive optics system of the Very Large Telescope. This demonstration presents the first online on-sky phase retrieval results ever obtained, and allows us to plan subsequent refinements on a well-tested basis. Among the potential refinements, and within the framework of high-contrast imaging of extra-solar planetary systems (which requires exquisite wavefront quality), we introduce an extension of the generalized NZ to the high-dynamic range case, and particularly to its use with the vector vortex coronagraph. This induces conjugated phase ramps applied to the orthogonal circular polarizations, which can be used to instantaneously retrieve the complex amplitude of the field, yielding a real-time calibration of the wavefront that does not need any other modulation such as focus or other deformable mirror probe patterns. Paper II (Riaud et al. 2012, A\&A, 545, A151) presents the mathematical and practical details of the new method.
\end{abstract}

Key words. instrumentation: high angular resolution - instrumentation: adaptive optics - methods: numerical techniques: polarimetric

\section{Introduction}

We present a phase retrieval method derived from the Nijboer-Zernike (NZ) theory of diffraction (i.e. the polar theory of diffraction) to sense optical aberrations in an imaging system. Sensing and subsequent correction, which is out of the scope of this paper, is crucial for optimizing the efficiency of imaging instruments. Within the framework of high-contrast imaging of extra-solar planetary systems, high-accuracy measurement and correction of wavefront is absolutely necessary for space-based or ground-based instruments. The current most popular phase diversity approach uses as its iterative-transform error-reduction method the Gerchberg-Saxton algorithm (Gerchberg \& Saxton 1972; Feinup 1982). Examples of application of this phase retrieval method are numerous: NAOS-CONICA (NACO), the adaptive optics system of the Very Large Telescope, the phasing of the James Webb Space Telescope (JWST), Palomar and Keck image-sharpening procedures, pre-correction of the NASA JPL High Contrast Imaging Testbed (HCIT), etc. Despite its success and widespread use, this technique has fundamental flaws:

- The error-reduction method heavily relies on Fourier transform, and in particular on its fast Fourier transform (FFT) implementation, which is fundamentally limited by numerical errors (sampling, wrap-arounds, round-off, etc.).
The NZ theory is fully analytical and relies on rigorous modal decomposition of aberrations.

- High-order aberration retrieval is generally noisy. Dean \& Bowers (2003), Sect. 9, presented the limitations of the classical error-reduction method, which heavily depends on the diversity nature and amplitude. The NZ theory overcomes this problem because it uses a modal description of the aberration directly at the focal plane.

- For a coupled amplitude and phase aberrations, the true wavefront is not retrieved by the iterative-transform method. Indeed, this generally does not take the amplitude error into account in the process. The NZ polar theory of diffraction uses complex $\beta_{n}^{m}$ coefficients that include both amplitude and phase aberrations.

In summary, the NZ phase retrieval approach, while generally using a focus modulation for diversity similar as the aforementioned method, allows us to retrieve the true input wavefront within a rigorous mathematical and analytical framework.

The first part of this first introductory paper presents the NZ-based phase retrieval technique and applies it to estimate non-common path aberrations of the adaptive optics NACO on the VLT. Note that this live demonstration is to our knowledge the first successful attempt at retrieving the phase online and 
on-sky, using real starlight instead of static and close-to-perfect calibration fibers.

We use this successful demonstration as a firm foundation to qualitatively introduce a novel instantaneous phase retrieval method finely and rigorously adapted from the NZ-based method to the vector vortex coronagraph (VVC). In this two-part presentation, we use the analogy between the focus modulation and the new photon orbital angular momentum (POAM) modulation provided by the novel coronagraphic device.

In Sect. 2, a description of the phase diversity concept using the NZ theory is given along with the specific algorithm. In Sect. 3, a presentation of the experimental validation of the NZ phase diversity is proposed with the NACO instrument on the VLT. Finally, in Sect. 4, we present a new concept of phase retrieval derived from the previous one, but using the VVC. Detailed explanations of the full theoretical and optical implementation with the VVC are presented in (Riaud et al. 2012, hereafter Paper II).

\section{Phase diversity using the NZ theory}

\subsection{The Nijboer-Zernike theory of diffraction}

The expression of the complex amplitude in the image plane $U(r, \phi)$ as a function of the pupil complex amplitude $P(\rho, \theta)$ can be calculated by the Fraunhofer diffraction integral in polar coordinates:

$U(r, \phi)=\frac{1}{\pi} \int_{0}^{1} \int_{0}^{2 \pi} P(\rho, \theta) \mathrm{e}^{(-2 \mathrm{i} \pi r \rho \cos (\theta-\phi))} \mathrm{d} \theta \rho \mathrm{d} \rho$.

The integration limits are defined by the pupil function $r \in[0,1]$, $\theta \in[0,2 \pi]$. We chose the diffraction integral independent of the wavelength, because our proposed modal decomposition will be adjusted with respect to the image sampling. The pupil complex amplitude $P(\rho, \theta)$ can be expressed in terms of a sum of Zernike polynomials $Z_{n}^{m}$ (Magette 2010 ):

$P(\rho, \theta)=\sum_{n, m} \beta_{n}^{m} Z_{n}^{m}(\rho, \theta) \quad \beta_{n}^{m} \in C$.

The $\beta_{n}^{m}$ are complex weighting coefficients, and the Zernike polynomials $Z_{n}^{m}(\rho, \theta)$ are defined as usual.

This polar diffraction theory, which expresses optical aberrations directly in the form of Zernike polynomials, is called Nijboer-Zernike (NZ) theory (Nijboer 1943, 1947). It is completely described in Magette (2010). Using explicit Bessel series to represent the diffraction integral (Janssen 2002), the NZ theory allows us to compute the final expression of the image intensity $I(r, \phi)$ thanks to a modal set of functions $V_{n, m}$, defined as follows:

$V_{n, m}(r)=(-1)^{(n+m) / 2} \frac{J_{n+1}(2 \pi r)}{2 \pi r} \quad\{n, m\} \in[0,+\infty]$

$I(r, \phi) \propto\left|\sum_{n, m} V_{n, m}(r)\left(\beta_{c n}^{m} \cos (m \phi)+\beta_{s n}^{m} \sin (m \phi)\right)\right|^{2}$,

where $\beta_{c n}^{m}, \beta_{s n}^{m}$ are coefficients for cos and sin functions respectively. In conclusion, the generalized NZ diffraction theory analytically computes the point spread function (PSF) of any optical system from its known pupil-plane aberrations, described by Zernike coefficients.

\subsection{The $N Z$ theory with defocus}

Estimating aberrations from the focused image alone does not ensure the uniqueness of the solution. This indetermination is due to the Fourier relationship between the PSF and the aberrated input phase: an infinite number of phase solutions exists in the pupil plane, which results in the same PSF. The classical phase diversity method (Blanc et al. 2003) introduces a known aberration (generally a defocus) to remove this indetermination (see Fig. 1).

Here we present a modified version of the NZ diffraction theory that allows taking into account a defocus term $f$ in the final image intensity. During the phase diversity process, the input Zernike polynomials are defocused and the polar transform becomes

$U(r, \phi, f)=\frac{1}{\pi} \int_{0}^{1} \int_{0}^{2 \pi} \mathrm{e}^{\mathrm{i} f \rho^{2}} Z_{n}^{m} \mathrm{e}^{(-2 \mathrm{i} \pi r \rho \cos (\theta-\phi))} \mathrm{d} \theta \rho \mathrm{d} \rho$

$I(r, \phi, f) \propto\left|\sum_{n, m} V_{n, m}^{f}(r)\left(\beta_{c n}^{m} \cos (m \phi)+\beta_{s n}^{m} \sin (m \phi)\right)\right|^{2}$.

The amplitude is the same as in the classical NZ theory, but using a new set of modal functions $V_{n, m}^{f}$ that take into account the focus amplitude $f$ :

$V_{n, m}^{f}(r)=(-1)^{m} \mathrm{e}^{\mathrm{i} f} \sum_{l=1}^{\infty} v_{f}(l, j) \frac{J_{m+l+2 j}(2 \pi r)}{l(2 \pi r)^{l}}$

$v_{f}(l, j)=(-2 \mathrm{i} f)^{l-1} \sum_{j=0}^{(n-m) / 2}(-1)^{(n-m) / 2} B(n, m, j, l)$

$B(n, m, j, l)=(m+l+2 j) \frac{\left(\begin{array}{c}m+j+l-1 \\ l-1\end{array}\right)\left(\begin{array}{c}j+l-1 \\ l-1\end{array}\right)\left(\begin{array}{c}l-1 \\ (n-m) / 2-j\end{array}\right)}{\left(\begin{array}{c}(n+m) / 2+l+j \\ l\end{array}\right)}$.

Physically, using the focus term $f$ induces a radial modulation of images with $f / 2 \pi$ nodes. The image intensity becomes

$$
\begin{aligned}
I(r, \phi)= & 4\left(\beta_{0}^{0}\right)^{2}\left|V_{0,0}\right|^{2}+f^{(1)}\left[\beta_{c n}^{m}, \beta_{s n}^{m}\right] \\
& +f^{(2)}\left[\left(\beta_{c n}^{m}\right)^{2},\left(\beta_{s n}^{m}\right)^{2},\left(\beta_{c n}^{m} \beta_{s n}^{m}\right)\right] .
\end{aligned}
$$

The intensity is composed of three different terms:

- $4\left(\beta_{0}^{0}\right)^{2}\left|V_{0,0}\right|^{2}:$ a constant term

- $f^{(1)}\left[\beta_{c n}^{m}, \beta_{s n}^{m}\right]$ : a linear function of $\beta_{n}^{m}$ coefficients

- $f^{(2)}\left[\left(\beta_{c n}^{m}\right)^{2},\left(\beta_{s n}^{m}\right)^{2},\left(\beta_{c n}^{m} \cdot \beta_{s n}^{m}\right)\right]$ : a term quadratic in the $\beta_{n}^{m}$ coefficients and $\cos /$ sin cross terms.

In the two functions $f^{(1)} / f^{(2)}$, the summation over $n, m$ values except $n=0, m=0$ are included. The complete mathematical development of $I(r, \phi)$ is presented in Paper II (Riaud et al. 2012).

\subsection{The $N Z$ phase retrieval process}

The NZ theory gives us tools for a straightforward and rigorous retrieval procedure. The first step of the retrieval process, which can be considered as a linearization, assumes that images can be described as linear combinations of the entrance pupil aberrations ( $\beta_{n}^{m}$ coefficients). This is equivalent to omitting $f^{(2)}$ terms. Equation (6) thus becomes

$I_{L}(r, \phi) \approx 4\left(\beta_{0}^{\prime 0}\right)^{2}\left|V_{0,0}\right|^{2}+f^{(1)}\left[\beta_{c n}^{m}, \beta_{s n}^{m}\right]$. 


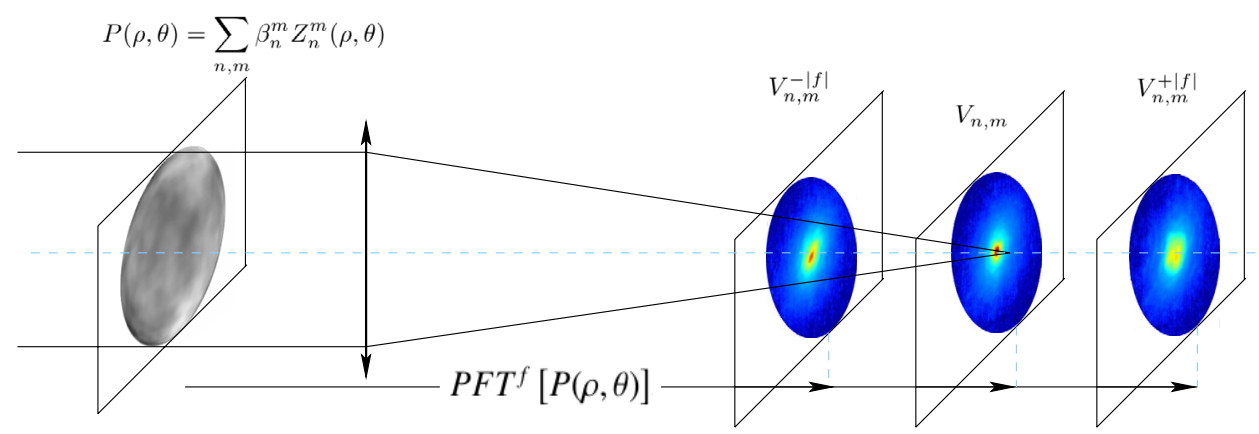

Fig. 1. Phase diversity principle. An entrance-aberrated pupil $P(\rho, \theta)$ is propagated through an optical system. For a circular pupil, the Fourier propagation between the pupil and the image plane is related to the polar Fourier transform equation $P F T[P(\rho, \theta)]$. If there is a focus aberration, this propagation must be developed as a function of the focus $f$ coefficient: the new optical propagation function becomes $P F T^{f}[P(\rho, \theta)]$. This mathematical development allows us to express the three image planes by a complete set of modal functions $V_{n, m}^{f}$.

The phase retrieval process based on the NZ theory then proceeds as a two-step procedure, filling both sides of an equality to subsequently identify them using the $\beta_{n}^{m}$ coefficient as free parameters. On one hand, we use the linearized modal expression the intensity PSF that we derived above, where a radial modal analysis was performed to analytically decompose the PSF into radial modes. On the other hand, we project the measured defocused PSFs on the basis of the same decoupled radial modes by means of a polar Fourier transform. The equalization of both sides of this newly formed equation (on one side we have an analytical model and on the other side the data) leads to the formations of decoupled systems of linear equations. Resolving these systems yields the aberration coefficients that we are looking for, and hence a first linear estimate of the wavefront.

In other words, analyzing the measured and model PSF with different foci on the basis of template modes $V_{n, m}^{f}$ leads indeed to a system of decoupled linear equations (Magette 2010), which after solving through classical matrix algebra calculation yields an estimate (an upper limit) of the aberrated coefficients $\beta_{n}^{m}$.

The quadratic term $f^{(2)}$ can then be directly calculated using the retrieved coefficients obtained in the first linearized step, using a recursive corrector approach defined by the following equations:

$$
\begin{gathered}
I(r, \phi)-f^{(2)}\left[\left(\beta_{c n}^{\prime m}\right)^{2},\left(\beta_{s n}^{\prime m}\right)^{2},\left(\beta_{c n}^{\prime m} \beta_{s n}^{\prime m}\right)\right]=I_{L}(r, \phi) \\
f^{(2)}\left[\left(\beta_{c n}^{\prime m}\right)^{2},\left(\beta_{s n}^{\prime m}\right)^{2},\left(\beta_{c n}^{\prime m} \beta_{s n}^{\prime m}\right)\right]=I(r, \phi)-I_{L}(r, \phi) .
\end{gathered}
$$

After iterating, $\beta_{n}^{\prime m}$ will tend to $\beta_{n}^{m}$ if and only if $f^{(2)}<f^{(1)}$.

\section{Application of classical NZ phase retrieval on NACO}

To illustrate the phase diversity retrieval using the NZ theory, we present an experiment recently conducted on-sky with NAOS-CONICA, the adaptive optics camera of the Very Large Telescope. Using CONICA, three focus images (i.e. images intra, in, and extra focus) of the star HD 25026 were taken on September 26, 2009 (prog. ID 383.C-0550(A)). We note that the images were taken in closed loop, using NAOS's visible wavefront sensor (Fig. 2). All images consist of a coaddition of 160 frames of $0.35 \mathrm{~s}$ exposure. Their observational characteristics and the setup are summarized in Table 1.

We then applied the classical NZ phase retrieval method presented above using these temporally averaged images of HD 25026. Our goal was to derive the complex amplitude of the NACO pupil online, in quasi real-time.

\subsection{Practical considerations}

The pixel sampling has to be adjusted at the beginning of the NZ retrieval process to take into account the tip-tilt blurring caused by the long exposure time (56 s). Indeed, with a camera sampling of 13.27 mas/pixel and an undersized Lyot stop inside the CONICA camera, the image sampling would be $\approx 4.67$ pixels $/(\lambda / D)$. The best sampling retrieved as a free parameter by the NZ analysis is 5.3 pixels $/(\lambda / D)$ (see Table 2 ). The difference of 0.62 pixel on the PSF width corresponds to 8.3 mas of PSF blurring, which is close to the estimated value of the tiptilt residuals. In other words, during a long exposure image, the turbulence increases the PSF width (blurring) and the NZ phase retrieval allows us to retrieve the long-term residual aberrations on the NACO instrument under sky operation.

One of the input parameters required by the NZ phase retrieval is a first estimate of the maximum number of Zernike polynomials that can be retrieved from the input sky images, which are indeed affected by the photon and the readout noises. Several retrieval runs with different numbers of complete Zernike coefficient sets $(21,28,36,45,55,66)$ empirically showed that using an optimal number of Zernike polynomials equal to 45 leads to a smaller $\beta_{n}^{m}$ coefficient variation. Indeed, if the number of Zernike coefficients is lower than the optimal value, fewer high frequencies can be retrieved. If the number of the Zernike aberrations is greater than the maximum limited by the input image's signal-to-noise ratio, the variance of the $\beta_{n}^{m}$ coefficients increases. The variance minimum of $\beta_{n}^{m}$ as a function of the number of Zernike coefficients indicates the highest spatial frequencies retrievable.

\subsection{Online phase retrieval results}

Figure 3 shows the retrieved aberrated pupil of NACO after our NZ phase retrieval while Fig. 4 gives the $\beta_{n}^{m}$ coefficient values. We note that the amplitude (top left in Fig. 3) is larger than 1, which is an artifact related to a global energy normalization in the PSF. We also note that amplitude variations are small, which indicates that very small amplitude aberrations (reflection or transmission problems) are present in the system.

Hartung et al. (2003) presented results of classical phase diversity on NACO using a fibered calibration source. Here, we used instead the direct on-sky image of a real star in closed-loop 

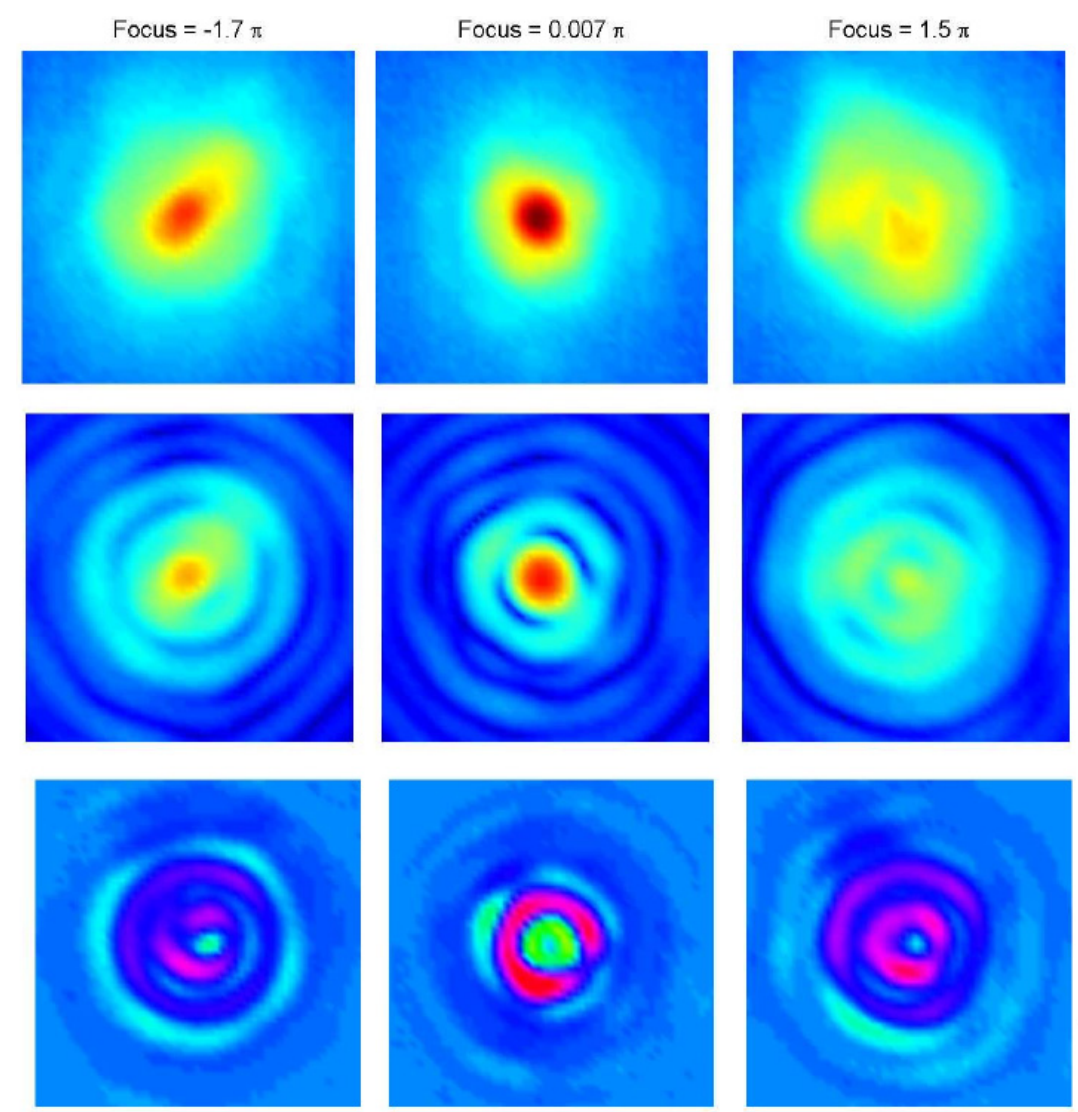

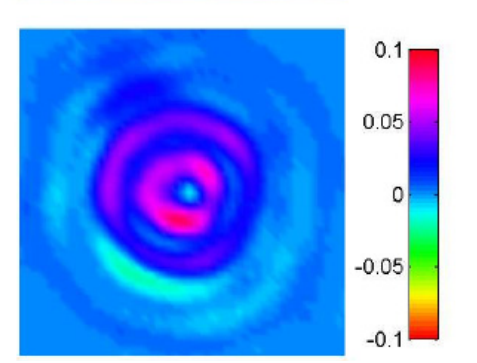

Fig. 2. Top: input PSFs used for the retrieval. Middle: output PSFs computed from the NZ phase retrieval. They are very similar to the input images in spite of the exposure time difference between the three defocused images. Bottom: differences between the input and retrieved PSFs. The low residuals $(<10 \%)$ are due to a blurring effect related to the tip/tilt during exposure time. The intensities are represented to the power $1 / 4$ to improve the contrast of the images.
Table 1. Characteristics of the images that were used to perform the phase retrieval.

\begin{tabular}{lc}
\hline \hline Star: HD 25026 & $m_{V}=9 m_{K}=4.93$ \\
\hline Resolution [mas/pix] & $13.27(\mathrm{~S} 13)$ \\
Number of exposures & 160 \\
Exposure time $[\mathrm{s}]$ & 0.35 \\
Wavelength $[\mu \mathrm{m}]$ and $R$ & $2.166\left(B_{\gamma}\right) R=70$ \\
Expected focus $f$ [mm] & $-3 / 0 /+3$ \\
NACO Lyot stop & "Undersize" 20\%-90\% \\
\hline
\end{tabular}

Table 2. Resuts of the NZ retrieval process.

\begin{tabular}{lc}
\hline \hline Sampling & $5.3 \mathrm{pix} /(\lambda / D)$ \\
\hline Intra focal position $[\mathrm{mm} / \mathrm{rad}]$ & $-3.32 /-1.7 \pi$ \\
Best focus position $[\mathrm{mm} / \mathrm{rad}]$ & $0.014 / 0.007 \pi$ \\
Extra focal position $[\mathrm{mm} / \mathrm{rad}]$ & $2.92 / 1.4 \pi$ \\
Number of Zernike polynomials & 45 \\
Strehl ratio without tip-tilt & $60 \%$ \\
Strehl ratio on the retrieved pupil & $36.5 \%$ \\
NACO Strehl ratio & $\approx 36-38 \%$ \\
\hline
\end{tabular}

(in the $B_{\gamma}$ filter, centered at $2.166 \mu \mathrm{m}$ ). The stellar averaged PSF is blurred due to tip-tilt and other high-order turbulence-induced aberrations uncorrected by the AO system, but it also presents clear underlying static residual phase and amplitude aberrations of about 0.105 wave rms $(\approx 60 \%$ Strehl ratio) for the phase and $\approx 10 \%$ error on the amplitude, respectively. The level of static amplitude aberrations measured on-sky could be the direct hint of an important item in the error budget that accounts
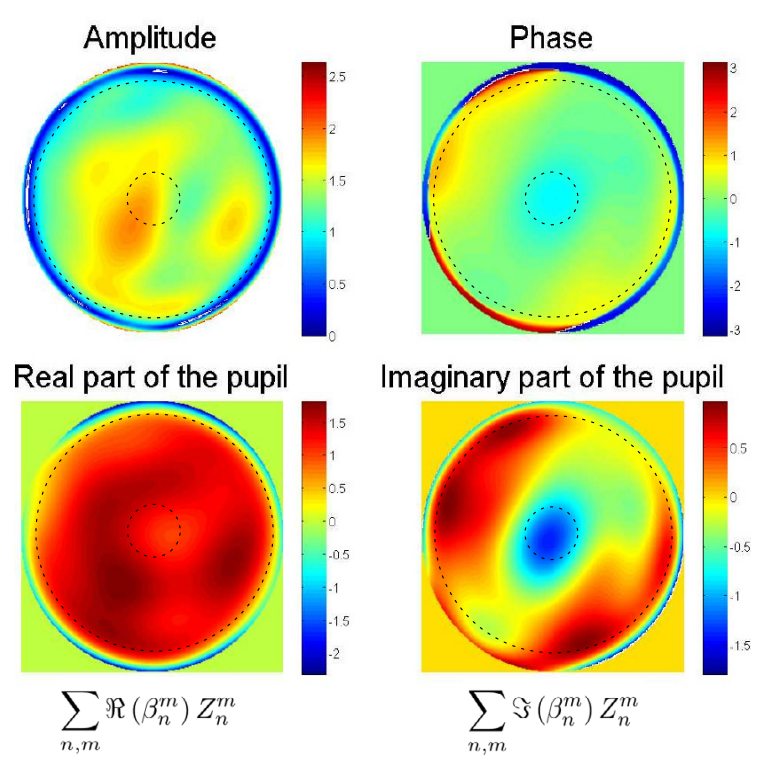

Fig. 3. Top: amplitude and phase calculated from the retrieved NZ aberrations. Bottom: real and imaginary parts of NACO's complex pupil. All images present border effects due to the "Undersized" diaphragm of the NACO coronagraph (the two dashed black circles in each image). The effect of the central obscuration is also visible, especially in the imaginary part of the pupil (bottom right).

for the actual Strehl limitation of NACO when observing on-sky. With the nominal calibration procedure of static aberrations described in Hartung et al. (2003), the unique DM will be driven to compensate for the phase but not the amplitude error of the wavefront. 
P. Riaud et al.: Nijboer-Zernike phase retrieval for high contrast imaging

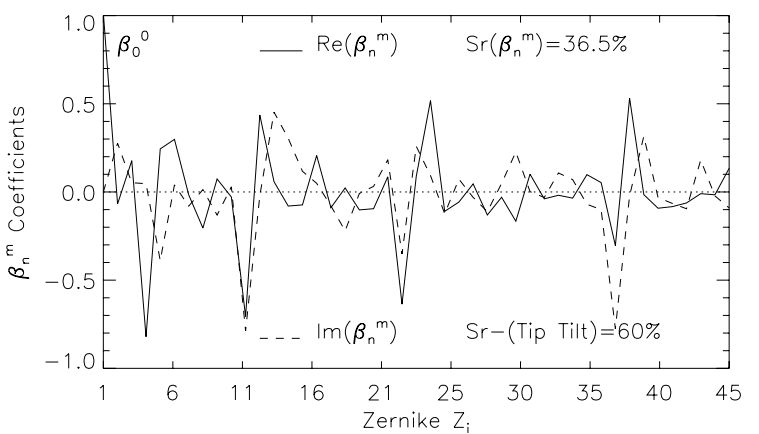

Fig. 4. $\beta_{n}^{m}$ coefficients values (real part as solid line and imaginary part a dashed line) on the first 45 Zernike polynomials. $\beta_{0}^{0}=1.0023$ and the retrieved Strehl ratio on the NACO diaphragm is $36.5 \%$ (see Table 2), very close to the estimated Strehl ratio provided by NAOS (36-38\%).

\section{Perspectives: NZ phase diversity with the VVC}

In this section, we present a promising evolution of the NZ phase retrieval method using the modulation properties of the VVC, which potentially would allow one to retrieve the phase instantaneously on-sky, requiring no offline (nor online) scanning in focus or any other kind of temporal wavefront modulation. The VVC indeed provides a natural instantaneous phase diversity that can be substituted for the classical focus variation. Using POAM modulation provided naturally by the VVC yields two significant advantages over the classical phase diversity: high dynamic range because the Airy pattern is naturally removed by the coronagraph, and instantaneity, because no scanning in focus or any other kind of temporal wavefront modulation is necessary.

\subsection{The vector vortex coronagraph}

The VVC is a transparent phase-mask that applies two conjugated phase ramps $\mathrm{e}^{\mathrm{i} \pm l_{\mathrm{p}} \phi}$ to the orthogonal circular polarization components of the incoming starlight, with $l_{\mathrm{p}}$ the topological charge or the POAM. When the VVC is centered on the PSF, it redirects the light outside the downstream pupil where it can be blocked by a Lyot stop (Mawet et al. 2005). Because of optical aberrations in the input pupil $P(\rho, \theta)$, the coronagraphic rejection (the stellar flux attenuation coefficient) is not perfect and a small amount of the starlight remains in the coronagraphic pupil plane $P_{\mathrm{c}}(\rho, \theta)$ after the diaphragm filtering by the Lyot stop (see Fig. 4). This residual light in turn focuses into aberrated PSFs, carrying the information about the aberrations modulated by the conjugated phase ramps.

\subsection{New modal decomposition for the NZ theory}

Here we establish the fundamental equation of the new enhanced NZ theory applied to the VVC-modulated PSFs. Note that the complete theoretical demonstration of VVC function properties under polar diffraction analysis (Fraunhofer and Fresnel propagation) is given in the more detailed Paper II (Riaud et al. 2012). We showed that going from the pupil to the first focal plane with a polar Fourier transform is $\operatorname{PFT}[P(\rho, \theta)]$, yielding the classical modal decomposition basis $V_{n, m}$. Multiplying by the VVC phase function, and performing the inverse polar transform to propagate to the downstream pupil yields $P F T^{l_{p},-1}[U(r, \phi)]$. Note that the remaining light in the coronagraphic pupil $P_{\mathrm{c}}(\rho, \theta)$ possesses a POAM $\pm l_{\text {p }}$ added by the phase-mask. The final polar transform
$\operatorname{PFT}^{l_{\mathrm{p}}}\left[P_{\mathrm{c}}(\rho, \theta)\right]$ to the final focal plane leads to a new modal decomposition using $V_{n, m}^{l_{\mathrm{p}}}$ functions defined as

$$
\begin{aligned}
& U\left(r, \phi, l_{\mathrm{p}}\right)=\frac{1}{\pi} \int_{0}^{d_{\mathrm{c}}} \int_{0}^{2 \pi} \mathrm{e}^{\mathrm{i} \mathrm{l}_{\mathrm{p}} \theta} Z_{n}^{m} \mathrm{e}^{(-2 \mathrm{i} \pi r \rho \cos (\theta-\phi))} \mathrm{d} \theta \rho \mathrm{d} \rho \\
& I\left(r, \phi, l_{\mathrm{p}}\right) \propto\left|\sum_{n, m} V_{n, m}^{l_{\mathrm{p}}}(r, \phi)\left(\beta_{c n}^{m} \cos (m \phi)+\beta_{s n}^{m} \sin (m \phi)\right)\right|^{2} \\
& V_{n, m}^{l_{\mathrm{p}}}(r, \phi)=\epsilon_{l_{\mathrm{p}}}(-1)^{(n+m) / 2} \mathrm{e}^{\mathrm{i} l_{\mathrm{p}} \phi} \frac{J_{n+1}(2 \pi r)}{2 \pi r} \\
& \epsilon_{l_{\mathrm{p}}}=-i, l_{\mathrm{p}} \neq 0 \quad \epsilon_{l_{\mathrm{p}}}=1, l_{\mathrm{p}}=0 \quad d_{\mathrm{c}}<1,
\end{aligned}
$$

where $d_{\mathrm{c}}$ is the size in pupil unit of the Lyot stop in the coronagraphic pupil.

\subsection{The phase retrieval process with VVC}

The VVC naturally simultaneously adds two conjugated multiplicative $\operatorname{Exp}\left[ \pm \mathrm{i} l_{\mathrm{p}} \phi\right]$ terms on the $V_{n, m}$ modal function at the first focal plane on the orthogonal circular polarizations (left- and right-hand). The direct modified polar transform $\operatorname{PFT}_{m}^{l_{\mathrm{p}}}\left[P_{\mathrm{c}}(\rho, \theta)\right]$ then yields two overlapping final attenuated coronagraphic image functions of the $l_{\mathrm{p}}$ or POAM parameter.

If the orthogonal circular polarizations are split (with a polarizing beamsplitter, see Fig. 5), we can directly and simultaneously have access to the opposite POAM modulations. Similarly to the classical NZ phase retrieval theory, but this time using the POAM instead of the focus as the modulator, the coronagraphic attenuated images can be projected onto the modified $V_{n, m}^{-\left|l_{\mathrm{p}}\right|}$ and $V_{n, m}^{+\left|l_{\mathrm{p}}\right|}$ modal functions.

The POAM phase retrieval process is the same as the classical focus phase diversity (with new modal functions, resolution of the linearized system, and iterative correction of the non-linear term), except that the $l_{\mathrm{p}}$ parameter is now fixed by the phase-mask. We note that the modulation that was radial with the focus diverstiy now becomes azimuthal with the VVC-POAM, but with $2 l_{\mathrm{p}}$ nodes.

In contrast to previous systems of phase retrieval using a purely recursive process, this novel technique is rigorous (analytical), and not limited to small aberrations. The simultaneous POAM modulation provided by the VVC allows us to extend the NZ theory to high dynamic coronagraphic images. The expected dynamical gain is twofold:

1 - reduced photon noise due to the coronagraphic attenuation of the Airy pattern;

2 - no radial modulation in the $V_{n, m}^{l_{\mathrm{p}}}$ functions that spread the final images when the focus is applied.

To additionally consolidate the validity of this new approach by analogy, we summarize in Table 3 the two phase retrieval modal decompositions in the NZ theory of diffraction, presented in this work, while emphasizing the equivalent role of focus and topological (or POAM).

A double modulation with $f$ and $l_{\mathrm{p}}$ is possible but more mathematically complicated. The focus modulation has to be introduced into the coronagraphic pupil (not in the entrance pupil) for the two $l_{\mathrm{p}}$ images. Theoretically, this process would allow an easy separation of the common and non-common path aberrations. 


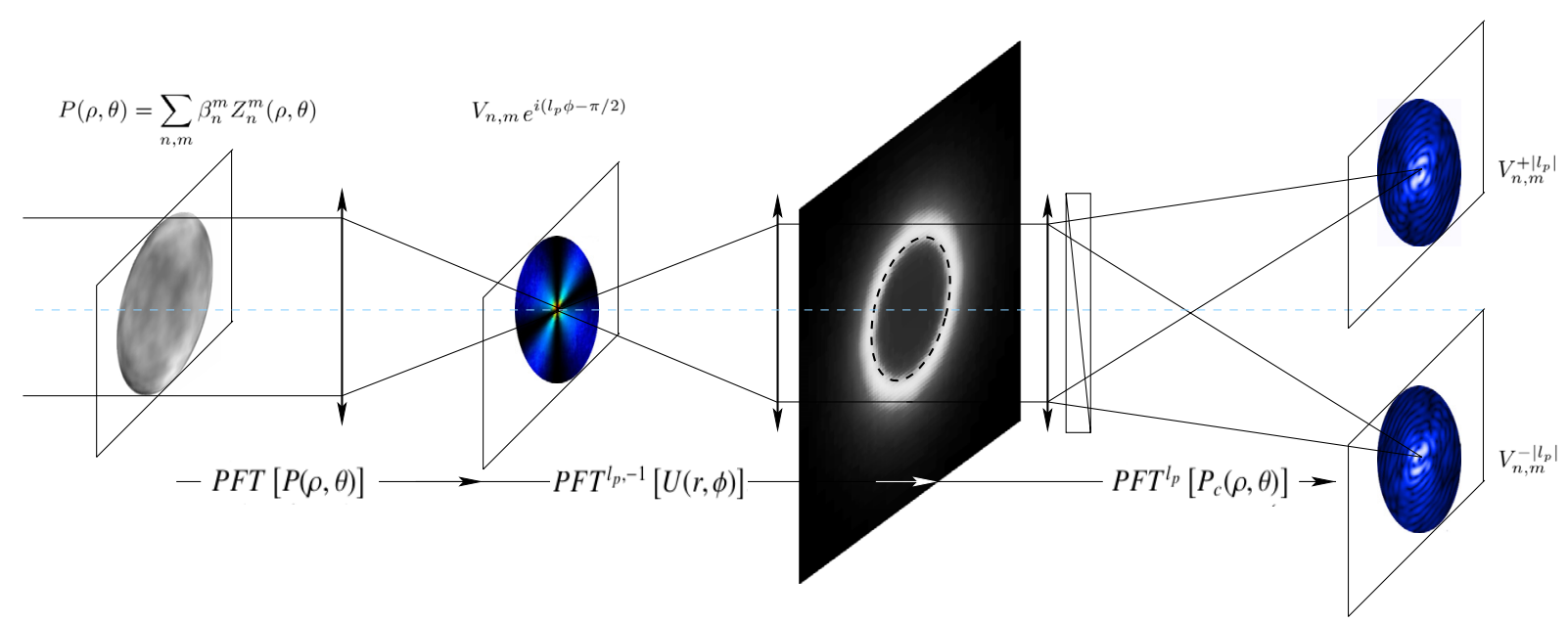

Fig. 5. POAM diversity principle. The optical propagation is more complicated for a coronagraphic phase-mask. Indeed, this scheme presents three different polar propagations with the same entrance aberrated pupil, which is presented in the classical phase diversity system. The first polar Fourier transform is the same as for classical imaging $(\operatorname{PFT}[P(\rho, \theta)])$, but after the phase-mask coronagraph, the inverse polar Fourier transform including the VVC POAM properties becomes $P F T^{\mathrm{p},-1}[U(r, \phi)]$. In the coronagraphic pupil plane $P_{\mathrm{c}}(\rho, \theta)$, the main part of stellar photons are rejected at the edge of the pupil and some of the remaining photons inside the pupil possess an added POAM $\pm l_{\mathrm{p}}$. The final polar propagation $P F T^{l_{\mathrm{p}}}\left[P_{\mathrm{c}}(\rho, \theta)\right]$ gives the final attenuated coronagraphic image. An optical polarizing system (quarter waveplates and Wollaston) located after the Lyot stop (the circular dashed circle in the coronagraphic pupil) allows us to separate the two circular polarizations and then the two POAMs. This mathematical development gives a complete set of modal functions $V_{n, m}^{l_{\mathrm{p}}}$.

Table 3. Different phase retrieval.

\begin{tabular}{lccc}
\hline \hline $\begin{array}{l}\text { Modal } \\
\text { functions }\end{array}$ & Parameter(s) & Modulation & Strehl \\
\hline$V_{n, m}^{f}$ & $\pm f$ & $r$ & $30-95 \%$ \\
$V_{n, m}^{l_{\mathrm{p}}}$ & $\pm l_{\mathrm{p}}$ & $\theta$ & $50-99+\%$ \\
$V_{n, m}^{f, l_{\mathrm{p}}}$ & $\pm f, \pm l_{\mathrm{p}}$ & $r, \theta$ & $50-99+\%$ \\
\hline
\end{tabular}

\section{Conclusion}

We presented the NZ phase retrieval approach to sense and calibrate wavefront aberrations (amplitude and phase). On-sky results under low Strehl ratio $(\approx 36-38 \%)$ conditions on the adaptive optics NAOS-CONICA were also presented as a proof of concept, showing great flexibility and ease of use for an online wavefront sensing methodology.

In the second part of this first paper, we introduced the extension of the NZ theory of diffraction to the high dynamic range case, and in particular to the VVC, which enables instantaneity of the phase retrieval process. Indeed, the VVC provides a natural instantaneous phase diversity that can be substituted for the classical focus variation. Using the POAM modulation provided naturally by the VVC yields two significant advantages over the classical phase diversity: high dynamic range because the Airy pattern is naturally removed by the coronagraph, and instantaneity, because no scanning in focus or any other kind of temporal wavefront modulation is necessary. For additional details and the thorough mathematical development of the new method, the interested reader is referred to our companion Paper II.

Acknowledgements. This work received the support of the University of Liège. The authors are grateful to C. Hanot (IAGL), and J. Surdej (IAGL) for the manuscript corrections. The authors wish to thank the referee Wesley Traub for useful comments and corrections. The authors also acknowledge support from the Communauté française de Belgique - Actions de recherche concertées Académie universitaire Wallonie-Europe. This idea dates back to 2005-2006 and the first author is grateful to Sect. 17 and the CNAP French commissions for their outstanding recruitment work.

\section{References}

Blanc, A., Fusco, T., Hartung, M., Mugnier, L. M., \& Rousset, G. 2003, A\&A, 399,373

Dean, B., \& Bowers, C. 2003, JOSA A, 20, 1490

Feinup, J. 1982, Appl. Opt., 21, 2758

Gerchberg, R., \& Saxton, W. 1972, Optik, 35, 237

Hartung, M., Blanc, A., Fusco, T., et al. 2003, A\&A, 399, 385

Janssen, A. 2002, JOSA A, 19, 849

Magette, A. 2010, Ph.D. Thesis: The International Liquid Mirror Telescope: optical testing and alignment using a Nijboer-Zernike aberration retrieval approch (IAGL, University of Liège), 1

Mawet, D., Riaud, P., Absil, O., \& Surdej, J. 2005, ApJ, 633, 1191

Nijboer, B. 1943, Physica, 10, 679

Nijboer, B. 1947, Physica, 13, 605

Riaud, P., Mawet, D., \& Magette, A. 2012, A\&A, 545, A151 